\title{
RNAi mediated silencing of Nanog expression suppresses the growth of human colorectal cancer stem cells
}

\section{Chen Zhang}

Minjiang University

\section{Yuanyuan Zhao}

Jilin University

\section{Yongjing Yang}

Cancer Hospital of Jilin Pronvince

Chunlian Zhong

Minjiang University

Tianju Ji

Jilin University

\section{Jinyue Duan}

Jilin University

Yi Wang ( $\nabla$ room1129@163.com )

Jilin University https://orcid.org/0000-0003-4021-1067

\section{Research article}

Keywords: Colorectal cancer, Cancer stem cells, Nanog, Proliferation, Apoptosis, Tumor growth

Posted Date: May 26th, 2020

DOl: https://doi.org/10.21203/rs.3.rs-22167/v1

License: (c) (i) This work is licensed under a Creative Commons Attribution 4.0 International License. Read Full License

Version of Record: A version of this preprint was published at Biochemical and Biophysical Research Communications on January 1st, 2021. See the published version at https://doi.org/10.1016/j.bbrc.2020.11.101. 


\section{Abstract}

Background: Colorectal cancer (CRC) is the third most common cancer in the world known for its poor recurrence-free prognosis. Previous studies have shown that it is closely linked with cancer stem cells (CSCs), which have self-renewal potential and the capacity to differentiate into diverse populations. Nanog is an important transcription factor that functions to maintain the self-renewal and proliferation of embryonic stem cells; however, many recent studies have shown that Nanog is also highly expressed in many cancer stem cells.

Methods: To investigate whether Nanog plays a crucial role in maintaining the stemness of colorectal CSCs (CCSCs), RNA interference was used to downregulate Nanog expression in the CRC stem cell line, $\mathrm{EpCAM}^{+} \mathrm{CD} 44^{+} \mathrm{HCT}-116$. We examined the anti-tumor function of Nanog in vitro and in vivo, using small interfering RNA.

Results: Our results revealed that the Nanog mRNA expression level in CCSCs was higher than that in HCT-116 cells. We found that the depletion of Nanog inhibited proliferation and promoted apoptosis in $\mathrm{EpCAM}^{+} \mathrm{CD} 44^{+} \mathrm{HCT}-116$ cells. In addition, the invasive ability of EpCAM ${ }^{+} \mathrm{CD} 44^{+} \mathrm{HCT}-116$ cells was markedly restricted when Nanog was silenced by small interfering RNA. Furthermore, we found that the silencing of Nanog decreased tumor size and weight and improved the survival rate of tumor-bearing mice.

Conclusions: In conclusion, these findings collectively demonstrate that Nanog, which is highly expressed in CRC stem cells, is a key factor in the development of tumor growth, and it may serve as a potential marker of prognosis and a novel and effective therapeutic target for the treatment of CRC.

\section{Background}

Colorectal cancer (CRC) is one of the most common malignant neoplasms with poor prognosis and high frequency of recurrence [1], accounting for 600,000 mortalities per year worldwide. Tumor recurrence and metastasis to distant organs are the main factors for the high mortality and low survival rates [2]. Although the incidence of $\mathrm{CRC}$ has decreased, current treatments have serious side effects, with a recurrence rate of more than $50 \%$, mainly due to resistance to conventional chemotherapy drugs $[3,4]$.

Recent studies have shown that cancer stem cells (CSCs), which are present in many tumor tissues, are a subset of cancer cells with the ability to self-renew. CSCs are primarily implicated in tumor initiation, progression, metastasis, and relapse after therapy [5-9] as well as colonic carcinogenesis [10]. The ineffectiveness of current cancer treatments may be the result of increased resistance to CSCs [11]. Thus, it is vital to improve the current therapeutic strategies for CRC and find novel treatments to eradicate cancer stem cells.

Nanog is a unique homeobox transcription factor required to maintain the self-renewal and pluripotency of embryonic stem cells (ESCs). Recently, emerging evidences have demonstrated that Nanog is 
expressed in a variety of cancer cell lines and tissues, and is associated with aggressive tumors $[12,13]$. A number of studies have revealed that the Nanog is a biomarker of CSCs, regulating cancer progression [14], as well as playing an important role in proliferation, apoptosis, differentiation, and stress response in CSCs in many cancers such as cervical, breast, and bladder cancers [14-17]. Several lines of evidence have suggested that the expression of Nanog is closely related to tumorigenesis, tumor metastasis, and distant recurrence after treatment [18]. Nanog has been extensively examined in human colorectal tissues, indicating its key role in maintaining colorectal CSC (CCSC) status and evasive resistance to conventional chemotherapy [19-21]. However, the underlying role of Nanog in CSCs in CRC remains to be elucidated.

In our previous study, we found that Nanog mRNA expression in CCSCs was higher than that in CRC cells. In the present study, we used RNA interference technology to silence Nanog mRNA and to examine the effect of Nanog on CCSCs. The results showed that silencing of Nanog suppressed proliferation, invasion, and tumorigenesis, as well as induced apoptosis of CCSCs, thus laying a foundation for further studies on the biological characteristics of CCSCs. Our findings also suggest that Nanog could be a novel therapeutic target in CRC.

\section{Methods}

Cell lines and cell culture

\section{Human CRC (HCT)-116 cell line (Cell Bank, Chinese Academy of Sciences, Shanghai, China) was}

cultured in RPMI 1640 medium supplemented with $10 \%$ fetal bovine serum (FBS), penicillin $(100 \mathrm{U} / \mathrm{ml})$, and streptomycin $(100 \mathrm{ug} / \mathrm{ml})$. EpCAM ${ }^{+}$CD44 ${ }^{+} \mathrm{HCT}-$ 116 cells (CCSCs) were cultured in Dulbecco's modified Eagle's medium with Ham's F12 (DMEM/F12) containing $2 \% \mathrm{~B} 27,20 \mathrm{ng} / \mathrm{ml}$ basic fibroblast growth factor (bFGF), and $20 \mathrm{ng} / \mathrm{ml}$ epidermal growth factor (EGF) as we previously described [7]. The cells were cultured in an incubator at $37^{\circ} \mathrm{C}$ containing $5 \% \mathrm{CO}_{2}$. 


\section{Animals}

A total of 35 6-week-old female athymic BALB/c nude mice $(20 \pm 2 \mathrm{~g})$ were purchased from Beijing HFK Bioscience Co., Ltd. and housed in the Laboratory Animal Center at Jilin University. All animals bred in specific pathogen-free conditions, with $25 \pm 2{ }^{\circ} \mathrm{C}$ temperature, $55 \pm 5 \%$ humidity and a 12 -h light/ dark cycle as the same conditions previously described [22]. Animals had ad libitum access to water and mouse chow diet. An acclimation period of at least 1 week was implemented for all mice prior to use in experiments. All the procedures were approved by Animal Care Committee of Jilin University (No. 20190046) and performed according to the Jilin University Guidelines for Animal Research.

\section{Reagents and antibodies}

The RPMI 1640 and DMEM/F12 were bought from Gibco (Grand Island, NY, USA) and the bFGF and EGF were purchased from PEPROTECH (Rocky Hill, NJ, USA). Anti-human CD44-biotin and anti-human epithelial cell adhesion molecule (EpCAM) for magnetic-activated cell sorting (MACS), were both purchased from eBioscience (San Diego, CA, USA). Phycoerythrin (PE)-anti-human CD44 and fluorescein isothiocyanate (FITC)-anti-EpCAM (eBioscience) were used for flow cytometric analysis. Mouse antiNanog and anti-GAPDH antibodies (Biolegend, San Diego, CA, USA), mouse anti-MMP-9, mouse antiMMP-2, mouse anti-Bax, mouse anti-Bcl-2, rabbit anti-cleaved caspase-3, rabbit anti-TIMP-1 were purchased from Abcam (Cambrige, UK) using for western blotting.

\section{Magnetic activated cell sorting (MACS)}

As we previously described, the experiment of MACS was performed using a CELLection ${ }^{\text {TM }}$ Biotin Binder kit following the manufacturer's instructions [7].In brief, HCT-116 cells were collected and incubated with anti-human EpCAM-biotin at $4^{\circ} \mathrm{C}$ for $10 \mathrm{~min}$. Subsequently, the cells were labeled with dynabeads (500 $\mu$ l) for $20 \mathrm{~min}$ at $4^{\circ} \mathrm{C}$, and a magnet was used to obtain the labeled cells (EpCAM cells). EpCAM ${ }^{+}$cells were then incubated in releasing buffer (Dnase l; $16 \mu \mathrm{l}$ ) for $15 \mathrm{~min}$ at room temperature (RT) and collected. $\mathrm{EpCAM}^{+}$cells were incubated with anti-human CD44-biotin for $10 \mathrm{~min}$, and subsequently with dynabeads $(50 \mu l)$ for $20 \mathrm{~min}$. Target cells (EPCAM $\left.{ }^{+} \mathrm{CD} 44^{+} \mathrm{CCSCs}\right)$ were separated using a magnet after adding releasing buffer (Dnase l; $4 \mu \mathrm{l}$ ) into the cells and incubating for $15 \mathrm{~min}$ at RT. EpCAM ${ }^{+} / \mathrm{CD}_{4} 4^{+} \mathrm{CCSC}$ were freshly prepared for use.

\section{Silencing by siRNA transfection}

To inhibit Nanog expression in the EpCAM+ CD44+ HCT-116 cells (CCSCs), silencing by small interfering RNAs (siRNAs) was performed using riboFECT ${ }^{\mathrm{TM}}$ CP Transfection Kit (Ribobio, Guangzhou, China) according to the manufacturer's instructions. Nanog siRNA and its negative control siRNA were designed and synthesized by Riobobio (Guangzhou, China). Nanog siRNA sequence is 5'-

AACTATCCATCCTTGCAAA-3'. The CCSCs were first cultured in 6-well plates ( $10^{6}$ cells/well) in DMEM/F12 medium for $48 \mathrm{~h}$, and then transfected with $20 \mu \mathrm{M}$ Nanog siRNA or negative control siRNA. 
Cells that had not been transfected served as controls. After transfection for $48 \mathrm{~h}$, the cells were cultured for further evaluation.

\section{Flow cytometry}

The cells $\left(10^{6} /\right.$ tube) were washed twice with phosphate-buffered saline (PBS) and incubated with PEanti-human CD44 and FITC-anti-human EpCAM (appropriate dilution per antibody) at $4^{\circ} \mathrm{C}$ for 20 min. Subsequently, the cells were washed again with PBS twice. The labeled cells were analyzed using a flow cytometer (BD Biosciences).

\section{Serum-induced differentiation}

To induce differentiation, CCSCs were cultured in DMEM/F12 medium supplemented with 10\% FBS for 3 days. The results were observed using an inverted microscope.

\section{Single-cell colony formation}

EpCAM $^{+}$CD $44^{+} \mathrm{HCT}-116$ cells were seeded in DMEM/F12 medium at a density of 200 cells per well on 6well plates and cultured at $37^{\circ} \mathrm{C}$ for 3 weeks. The medium was replaced every 2 to 3 days. Plates were photographed with an optical inverted microscope.

\section{Quantitative real-time polymerase chain reaction (qRT-PCR)}

Total cellular RNA was extracted using TRIzol reagent (Invitrogen, USA); reverse transcription was performed from $1 \mu \mathrm{g}$ of the total RNA using PrimeScript RT Master Mix (TaKaRa, Dalian, China), according to the manufacturer's instructions. qRT-PCR was performed with TransStart Top Green qPCR SuperMix (TransGen, Beijing, China) using a real-time PCR system (PikoReal 96, ThermoFisher Scientific, USA) with the following program: $94^{\circ} \mathrm{C}$ for $30 \mathrm{sec}$, followed by 40 cycles of amplification $\left(94^{\circ} \mathrm{C}\right.$ for $5 \mathrm{sec}$, $60^{\circ} \mathrm{C}$ for $15 \mathrm{sec}$, and $72^{\circ} \mathrm{C}$ for $1 \mathrm{sec}$ ). The primer sequences (GeneCreate, Wuhan, China) used for quantitative real-time PCR are shown in Table 1. GAPDH was used as an endogenous control. The relative expression levels of mRNA transcripts were analyzed by the $2^{-\Delta \Delta C t}$ method. All the experiments were performed in triplicate.

\section{Western blot analysis}

The CCSCs transfected with Nanog siRNA or negative control siRNA were harvested $48 \mathrm{~h}$ after transfection. For western blot analysis, cells were lysed in radioimmunoprecipitation assay (RIPA) buffer containing a protease inhibitor (DINGGUO, Beijing, China). After quantification of protein concentrations using a BCA protein assay kit (Beyotime, Nanjing, Jiangsu, China), equivalent quantities of protein (30 $\mu \mathrm{g} /$ well) were separated by $12 \%$ sodium dodecyl sulfate-polyacrylamide gel electrophoresis (SDS-PAGE) and transferred to polyvinylidene fluoride (PVDF) membranes. The membranes were incubated in blocking solution ( $5 \%$ non-fat milk/TBST), and then incubated with the following primary antibodies overnight at $4^{\circ} \mathrm{C}$ : mouse anti-Nanog (1:1000), mouse anti-MMP-9 (1:500), mouse anti-MMP-2 (1:500), 
mouse anti-Bax (1:500), mouse anti-Bcl-2 (1:500), rabbit anti-cleaved caspase-3 (1:500), rabbit anti-TIMP1 (1:500) and mouse anti-GAPDH (1:2000). After being washed three times with TBST, the membranes were incubated with horseradish peroxidase (HRP)-conjugated secondary antibody for $1 \mathrm{~h}$ at RT. Then, the membranes were washed again three times with TBST. The protein bands were detected using enhanced chemiluminescence detection kit (ECL; Beyotime, Nanjing, Jiangsu, China) on ChemiDoc XPS system (Bio-Rad, USA), and GAPDH was used as the loading control. Protein expression was quantified using Image Lab 5.2.1 (Bio-Rad) and normalized to GAPDH.

\section{MTS cell proliferation assay}

Cell proliferation was assessed using CellTiter 96 Aqueous assay kit (Promega; Madison, WI, USA). The CCSCs transfected with Nanog siRNA or negative control siRNA were plated at a density of $10^{4}$ cells/well into 96 -well plates $(100 \mu \mathrm{l}$ medium/well). After 48 h, $40 \mu \mathrm{l}$ of 3-(4,5-dimethylthiazol-2-yl)-5-(3-Carboxymethoxyphenyl)-2-(sulfophenyl)-2H-tetrazolium solution (MTS) was added to each well according to the manufacturer's instructions, followed by incubation of the plates at $37^{\circ} \mathrm{C}, 5 \% \mathrm{CO}_{2}$ for $2 \mathrm{~h}$, and the absorbance was measured at $490 \mathrm{~nm}$ using a microplate reader. All experiments were performed in triplicate and repeated three times.

\section{Annexin $\mathrm{V}$ analysis}

Annexin V analysis was performed using the -Annexin V-FITC kit (KeyGEN, Nanjing, Jiangsu, China) according to the manufacturer's instructions. Briefly, after CCSCs were transfected with Nanog siRNA or negative control siRNA for $48 \mathrm{~h}$, they were harvested and washed twice with PBS and then re-suspended in binding buffer at a density of $1 \times 10^{6} \mathrm{cell} / \mathrm{s} / \mathrm{ml}$. Subsequently, Annexin V-FITC $(5 \mu \mathrm{l})$ and propidium iodide (PI) $(5 \mu \mathrm{l})$ were added to the cells $(500 \mu \mathrm{l})$. After being incubated for $15 \mathrm{~min}$ in the dark at RT, the cells were analyzed using a flow cytometer (BD Biosciences, USA). Annexin $\mathrm{V}^{-} / \mathrm{PI}^{-}$cells present cell survival, Annexin $\mathrm{V}^{+} / \mathrm{PI}^{-}$cells were shown cells in early apoptosis, and Annexin $\mathrm{V}^{+} / \mathrm{PI}^{+}$cells were in late apoptosis or necrotic. The experiments were repeated independently three times.

\section{JC-1 assay}

Alterations in mitochondrial membrane potential were measured by flow cytometry using the JC-1 kit (KeyGEN, Nanjing, Jiangsu, China) according to the manufacturer's instructions. Briefly, after CCSCs were transfected with Nanog siRNA or negative control siRNA for $48 \mathrm{~h}$, the cells were harvested and washed twice with PBS and re-suspended in $500 \mu$ incubation buffer containing the JC- 1 dye $(1 \mu \mathrm{l})$ at a density of $1 \times 10^{6} \mathrm{cells} / \mathrm{ml}$. After being incubated for $15 \mathrm{~min}$ at $37^{\circ} \mathrm{C}, 5 \% \mathrm{CO}_{2}$, the cells were collected and washed twice with the incubation buffer. Subsequently, cells were re-suspended in $500 \mu l$ incubation buffer and analyzed using a flow cytometer (BD Biosciences, USA). Annexin $\mathrm{V}^{-} / \mathrm{Pl}^{-}$cells present cell survival, Annexin $\mathrm{V}^{+} / \mathrm{Pl}^{-}$cells were shown cells in early apoptosis, and Annexin $\mathrm{V}^{+} / \mathrm{PI}^{+}$cells were in late apoptosis or necrotic. The experiments were repeated independently three times. 


\section{Transwell invasion assay}

The invasion assays were performed using $6.5-\mathrm{mm}$ diameter Transwell plates $(8 \mu \mathrm{m}$ pore size, Corning, Steuben County, NY, USA) coated with a thin layer of Matrigel (BD Biosciences, San Diego, CA, USA), through which invading cells could migrate and eventually attach to the bottom of the polycarbonate layer. The CCSCs were resuspended in serum free DMEM/F12 at a concentration of $1 \times 10^{6} / \mathrm{ml}$. The upper chamber was loaded with $100 \mu \mathrm{l}$ of cell suspension and the lower chamber was loaded with $500 \mu \mathrm{l}$ of DMEM/F12 medium supplemented with $10 \%$ FBS as the chemoattractant. After incubation for $24 \mathrm{~h}$ at $37^{\circ} \mathrm{C}$ and $5 \% \mathrm{CO}_{2}$, non-invading cells in the upper chamber were removed with a PBS-soaked cotton swab and the cells that had invaded the membranes were stained with $0.5 \%$ crystal violet and counted under a light microscope. Each assay was replicated 3 times. The invaded cells were counted under the microscope in five random fields in each chamber. The assay was performed in triplicate.

\section{In vivo tumor xenograft assay}

To generate tumor xenografts, EpCAM ${ }^{+} \mathrm{CD} 44^{+} \mathrm{HCT}-116$ cells $\left(5 \times 10^{5}\right)$ were injected subcutaneously into the right flank of each mouse. When the mice attained a tumor volume of $40-60 \mathrm{~mm}^{3}$, they were randomly divided into 3 groups $(n=10)$ and treated with Nanog siRNA, mock, or negative control siRNA. Nanog siRNA or negative control siRNA was injected intratumorally twice a week for 3 weeks. The tumor size was measured every other day using a vernier caliper and calculated $a s\left(a \times b^{2}\right) / 2$, where a is the tumor length and $b$ the width. At the end of the experiment, mice were euthanized by carbon dioxide asphyxiation for approximately $6 \mathrm{~min}$ (air displacement rate: 20\%/min; carbon dioxide flow rate: 1.7 $\mathrm{L} / \mathrm{min}$; the mortality was ensured by cervical dislocation) and tumors were excised and weighed.

\section{Statistical analysis}

All data are presented as the mean \pm standard deviation (SD) of at least three repeat experiments. Student's t-test and one-way ANOVA analysis were used to analyze the variances between groups. The log-rank test was used to compare the survival rates in different groups. Significant differences were considered when $\mathrm{P}$ values were less than 0.05 .

\section{Results}

\section{Screening and identification of CCSCs from HCT-116 CRC cell lines}

EpCAM and CD44 have been previously identified as surface markers for CCSCs and used for isolating CCSCs from CRC cells $[23,24]$. In our study, the proportion of the EpCAM ${ }^{+} \mathrm{CD} 44^{+}$subpopulation of HCT116 cells isolated by MACS accounted for $<2.00 \%$, and the CCSCs formed spheres after being cultured for 7 days in serum-free medium (Fig. 1A). The expressions of EpCAM and CD44 were evaluated by flow cytometry and the percentage of the EpCAM ${ }^{+} \mathrm{CD} 44^{+} \mathrm{HCT}-116$ cells in the sorted cells were significantly higher than that in unsorted HCT-116 cells (Fig. 1B). Subsequently, CCSC spheres were observed over 21 
days by the single-cell colony formation assay (Fig. 1C). CCSC spheroid cells became re-adherent and differentiated after serum was added to the medium (Fig. 1D).

\section{High expression level of Nanog in CCSCs, and siRNA-mediated knockdown of Nanog}

To address whether Nanog can serve as a novel therapeutic target for CRC, the relative mRNAs expression of Nanog, Sox-2, Oct-4, and C-myc as putative stem cell markers, were analyzed by real-time PCR. EpCAM ${ }^{+} C D 44^{+}$cells exhibited significantly higher relative Nanog and Oct-4 mRNAs level $(7.03 \pm 0.12$ and $4.37 \pm 0.18$, ) than HCT-116 cells $(1.00 \pm 0.066$ and $1.00 \pm 0.17)$ in Figure. $2 \mathrm{~A}$.

To investigate the function of Nanog in CCSCs, Nanog-specific siRNA was transfected into CCSCs (CCSCsiNanog), and its transfection efficiency was analyzed with a fluorescent microscope (Fig. 2B). Subsequently, its ability to downregulate Nanog expression was evaluated by RT-qPCR (Fig. 2C) and western blot analysis (Fig. 2D-E). There was a significant decrease in Nanog expression of mRNA or protein with Nanog-siRNA transfection (mRNA $0.23 \pm 0.070$, protein $0.33 \pm 0.012$ ) compared with mock (mRNA $1.00 \pm 0.0064$, protein $1.00 \pm 0.0083$ ) or negative control (mRNA $0.99 \pm 0.048$, protein $0.99 \pm 0.0078$ ) siRNA transfection, indicating that silencing of Nanog by the siRNA method was effective.

\section{Down-regulation of Nanog inhibits CCSC proliferation and promotes apoptosis}

To investigate the effect of Nanog on self-renewal of CCSCs, the MTS cell proliferation assay was performed. Proliferation ratio of CCSC-siNanog group (54.71 $\pm 8.01 \%$ ) was significantly inhibited compared with that of mock $(100.00 \pm 3.12 \%)$ or negative control groups $(96.73 \pm 5.72 \%)$ in Figure. $3 \mathrm{~A}$.

To further examine the inhibitory effects of Nanog on CCSCs proliferation, assessment of apoptosis was performed after transfection with siRNA. The Annexin V/PI assay revealed that compared with Mock (4.16\%) or NC siRNA (10.02\%) groups, Nanog-siRNA (68.47\%) transfection significantly increased the ratio of Annexin V-positive cells (Fig. $3 \mathrm{C}$ and D), at the meanwhile, the results of JC-1 staining assay indicated that the percentage of cells undergoing a loss of mitochondrial membrane potential increased significantly following Nanog siRNA (32.31\%) transfection compared with Mock (4.94\%) or NC siRNA (8.54\%) groups (Fig. 3E and F). These results suggest that silencing of Nanog promotes CCSC apoptosis. In addition, the expression of B-cell lymphoma 2 (Bcl-2), Bcl-2 associated X protein (Bax), and caspase-3 were evaluated using RT-qPCR and western blot analysis. After treatment with Nanog siRNA for $48 \mathrm{~h}$, the mRNA and protein expression of $\mathrm{Bcl}-2$ (mRNA $0.33 \pm 0.027$, protein $0.29 \pm 0.036$ ) was significantly suppressed while the mRNA and protein expressions of Bax (mRNA 6.07 \pm 0.82 , protein $5.50 \pm 0.041$ ) and cleaved-caspase-3 (mRNA $2.68 \pm 0.32$, protein $2.07 \pm 0.039$ ) were significantly up-regulated (Fig. 3B, $G$ and $\mathrm{H})$. Taken together, these results demonstrated that silencing of Nanog inhibited the proliferation of CCSCs and promoted significant apoptosis.

\section{Nanog silencing decreases the invasive ability of CCSCs in vitro}


In order to investigate the effect of Nanog on the invasive ability of CRC stem cells, which is often representative of the metastatic potential, the Transwell invasion assay was performed after the silencing of Nanog. Nanog siRNA (62 \pm 5$)$ significantly decreased the number of CCSCs that passed through the matrigel compared to those of mock $(143 \pm 3)$ or negative control siRNA $(140 \pm 4)$ (Fig. 4A and B).

Additionally, we analyzed the alterations in the expression of invasion-related genes by RT-qPCR and western blot. After transfection of Nanog siRNA for $48 \mathrm{~h}$, the mRNA and protein expression of matrix metalloproteinases, MMP-2 (mRNA 0.35 \pm 0.048 , protein $0.33 \pm 0.038$ ), and MMP-9 (mRNA 0.26 \pm 0.068 , protein $0.29 \pm 0.039$ ), that promote tumor invasion and metastasis, were significantly reduced. However, the expression of tissue inhibitor of metalloproteinases-1 (TIMP-1, mRNA 2.42 \pm 0.064 , protein $2.32 \pm 0.047$ ) was significantly increased in CCSC-siNanog compared with that in mock or negative control CCSCs (Fig. 4C-E).

The heat map (summarized the mRNA expression data of Bcl-2, Bax, caspase-3, MMP-2, MMP-9 and TIMP-1) revealed that Nanog silencing enhanced the expression of Bax, caspase-3, and TIMP-1 (Fig. 5, in red color), and decreased the expression of Bcl-2, MMP-2, and MMP-9 (Fig. 5, in blue color).

\section{Nanog siRNA treatment suppressed tumor growth in vivo and increased mice survival rate}

Based on the in vitro studies described above, we further investigated the effect of Nanog siRNA on tumor growth in vivo. A total of 35 of 6-week-old female athymic BALB/c nude mice ( $20 \pm 2 \mathrm{~g}$ ) were randomly divided into 3 groups ( $n=10$, note: 5 of mice were excluded because they were no obvious tumors after injected EpCAM ${ }^{+}$CD $44^{+} \mathrm{HCT}-116$ cells for 2 weeks) and treated with Nanog siRNA, mock, or negative control siRNA. After treatment with Nanog siRNA or negative control siRNA (injected intratumorally twice a week for 3 weeks), we found Nanog siRNA treatment strongly decreased tumor size and weight compared with negative control siRNA or mock treatments (Fig. 6A and B). Meanwhile, in comparison with the mock $(0 / 5$ mice were alive at day $48, n=5)$ or control groups $(0 / 5$ mice were alive at day $48, n=5)$, Nanog siRNA treatment ( $4 / 5$ mice were alive at day $48, n=5)$ significantly improved the survival rate of tumor-bearing mice (Fig. 6C). Taken together, these results demonstrate that Nanog siRNA treatment suppresses colorectal tumor growth in vivo and improves survival, implying that Nanog may serve as a novel therapeutic target for CRC treatment. For survival analysis, mice were sacrificed when tumors measured $>2000 \mathrm{~mm}^{3}$ or the tumor diameter exceeded $2.0 \mathrm{~cm}$. All of the treatments with mice are according to the protocol of the Animal Care Committee of Jilin University as previously described [22].

\section{Discussion}

In recent years, CRC has been ranked as the third leading cause of cancer-related mortalities among malignant tumors worldwide [25]. Advances in the investigation of cancer stem cells have revealed that CSCs have the ability to self-renewal and maintain tumor growth, and therefore play a crucial role in tumorigenesis, development, metastasis, and recurrence [5, 6]. Nanog has been established as an important transcriptional factor required for maintaining self-renewal and pluripotency of embryonic stem 
cells [25]. Previous studies have shown that Nanog is overexpressed in various types of CSCs [14-16] including CRC stem cells [20]. It is consistent with previous studies [26, 27], the CCSCs in the present study displayed relatively higher expression levels of Nanog compared with that in unsorted HCT-116 cells. Furthermore, emerging evidence has suggested that Nanog plays a vital role during self-renewal of CSCs, and knockdown or silencing of Nanog suppresses CSCs growth and development [28]. These results suggest that Nanog may be associated with tumor initiation, development, and therapeutic resistance. However, the role of Nanog in CRC stem cells has not been investigated.

To understand the functional role of Nanog in CCSCs, we used the RNA interference approach to knockdown Nanog expression in EpCAM ${ }^{+} \mathrm{CD} 44^{+} \mathrm{HCT}-116$ cells, and assessed its effect on suppressing growth and promoting apoptosis of CCSCs in vitro. Our results, which revealed that Nanog silencing not only significantly suppressed CCSC proliferation but also markedly induced CCSC apoptosis, ware similar to those of studies reported for pancreatic and breast cancer stem cells [21,29]. There are two main pathways associated with apoptosis: a pathway mediated by a cell death receptor and that mediated by mitochondrion, both of which lead to the activation of the caspase cascade. Mitochondrial membrane potential is regulated by a complex network of signaling pathways that involve endogenous pro- and antiapoptotic Bcl-2 family proteins [30]. The results of the Annexin $\mathrm{V}$ assays provided evidence that the depletion of Nanog promoted apoptosis of CCSCs, which is in accordance with the results of inhibition of Nanog and aggravation of apoptosis in non-small cell lung cancer [31]. Moreover, the results of JC-1 assays suggested that a loss in mitochondrial transmembrane potential $(\Delta \psi \mathrm{m})$ was observed in the Nanog siRNA group compared with that in the mock and negative control siRNA groups. In addition, we analyzed the mRNA levels and protein expression of several apoptosis-related genes since previous studies have shown that resistance to apoptosis is one of the leading causes of tumorigenesis $[8,16,32]$. Caspase- 3 is a downstream molecule that is activated by upstream molecules such as caspase- 8 or caspase-9, leading to cell apoptosis. Pro-apoptotic Bax promotes the release of pro-apoptotic molecules by forming oligomers in the mitochondrial outer membrane, thus promoting cell apoptosis. Anti-apoptotic $\mathrm{Bcl}-2$ blocks mitochondrial apoptosis by blocking the release and oligomerization of Bax [33]. Thus, Caspase-3 and the Bcl-2 family proteins play an important role in the regulation of apoptosis [34]. Nanog silencing activated cleaved caspase- 3 expression, promoted the expression of pro-apoptotic BAX gene, and inhibited the expression of anti-apoptotic $B C l-2$ gene. These results indicate that Nanog may play a vital role in the induction of apoptosis in CCSCs via the caspase-3 cascade.

It is well known that the invasive ability of CSCs is crucial for cancer metastasis [35]. Further, high expression levels of matrix metalloproteinases (MMPs), which are identified as potential major regulators of invasion, are associated with tumor progression and metastasis in diverse human cancers [33]. The tissue inhibitors of metalloproteinases (TIMPs), which are inhibitors of MMPs, have been shown to impede tumor progression [36]. In our study, Transwell invasion assay and analyses of mRNA and protein expressions of MMP-2, MMP-9, and TIMP-1, in line with the results of the regulation of MMP-2, 9, and TIMP-1, promote the invasion and metastasis of renal cell carcinoma and MMP-9 (TIMP-1 is its inhibitor) in the degradation of extracellular matrix. This enhances metastasis in breast cancer; thus revealing that 
Nanog silencing significantly suppressed the invasive potential of CCSCs $[37,38]$. Thus, Nanog may play a crucial role in the invasive potential of CSCCs [39]. Consistent with our in vitro results, in vivo results showed that Nanog siRNA significantly suppressed xenograft tumor growth and prolonged mice survival.

\section{Conclusions}

In conclusion, our results demonstrated that silencing of Nanog expression suppressed the proliferation, invasion, and tumorigenesis, as well as induced apoptosis of CCSCs in vitro and in vivo, all of which are crucial for cancer development. Furthermore, our findings revealed for the first time the key role of Nanog in tumor growth in CRC. Thus, Nanog-targeted siRNA may provide a possible novel strategy for cancer stem cell-based targeted therapies in CRC. The exact mechanism by which Nanog silencing exerts its antitumor ability may be worth exploring in future investigations.

\section{Abbreviation}

CRC, Colorectal cancer; CSCs, Cancer stem cells; CCSCs, Colorectal CSCs; ESCs, Embryonic stem cells; DMEM/F12, Dulbecco's modified Eagle's medium with Ham's F12; FBS, Fetal bovine serum; bFGF, Basic fibroblast growth factor; EGF, Epidermal growth factor; EpCAM, Epithelial cell adhesion molecule; MACS, Magnetic-activated cell sorting; PE, Phycoerythrin; FITC, Fluorescein isothiocyanate; RT, Room temperature; siRNA, Small interfering RNA; PBS, Phosphate-buffered saline; qRT-PCR, Quantitative realtime polymerase chain reaction; SDS-PAGE, Sodium dodecyl sulfate-polyacrylamide gel electrophoresis; PVDF, Polyvinylidene fluoride; HRP, Horseradish peroxidase; MTS, 3-(4,5-dimethylthiazol-2-yl)-5-(3-Carboxymethoxyphenyl)-2-(sulfophenyl)-2H-tetrazolium solution; $\mathrm{PI}$, Propidium iodide; MMPs, Matrix metalloproteinases; TIMPs, Tissue inhibitors of metalloproteinases; Bcl-2, B-cell lymphoma; Bax, Bcl-2 associated $X$ protein.

\section{Declarations}

Ethics approval and consent to participate

All experiments and animal care were conducted according to the guidelines established by the Animal Care Committee of Jilin University (Institutional Animal Care and Use Committee of Jilin University School of Pharmaceutical Science, Number of permit: 2019-0046).

Consent for publication

Not applicable.

Availability of data and material

Not applicable.

Competing interests 
The author(s) declare that they have no competing interests.

Funding

This work was supported by Jilin Province Science and Technology Support Program (Grant number: 20200404121YY), the Education Department of Jilin Province(Grant number:JJKH20201122KJ), Jilin Province Health Technology Innovation Project (Grant number: 2017J062), National Natural Science Foundation of China (Grant number: 81801849) and Talent Introduction Fund of Minjiang University (Grant number: MJY19011 and MYK19033). The funding body of 2017J062, 81801849, MJY19011 and MYK19033 designed the study, purchased the experimental materials, 20200404121YY collected and analyzed the data; JJKH20201122KJ interpreted the data; 20200404121YY, JJKH20201122KJ and MJY19011 supported in writing the manuscript.

Authors' contributions

C.Z. and Y.Z. conceived the experiment(s), Y.W. conducted the experiment(s), T.J. and J.D. performed the experiments. C.Z., Y.Z., Y.Y. and CL.Z. analyzed the results. C.Z. and Y.Z wrote the manuscript. All authors reviewed the manuscript.

Acknowledgments

Not applicable.

\section{References}

1. Figueredo AJ, Wolf PSA: Assortative Pairing and Life History Strategy A Cross-Cultural Study. Hum Nature-Int Bios 2009, 20(3):317-330.

2. Hao Z, AghaKouchak A, Nakhjiri N, Farahmand A: Global integrated drought monitoring and prediction system. Sci Data 2014, 1:140001.

3. Zisman TL, Rubin DT: Colorectal cancer and dysplasia in inflammatory bowel disease. World J Gastroenterol 2008, 14(17):2662-2669.

4. Cunningham D, Atkin W, Lenz HJ, Lynch HT, Minsky B, Nordlinger B, Starling N: Colorectal cancer. Lancet 2010, 375(9719):1030-1047.

5. Zhang N, Yin Y, Xu SJ, Chen WS: 5-Fluorouracil: mechanisms of resistance and reversal strategies. Molecules 2008, 13(8):1551-1569.

6. Jiang WQ, Fu FF, Li YX, Wang WB, Wang HH, Jiang HP, Teng LS: Molecular biomarkers of colorectal cancer: prognostic and predictive tools for clinical practice. J Zhejiang Univ Sci B 2012, 13(9):663675.

7. Zhang C, Tian Y, Song F, Fu C, Han B, Wang Y: Salinomycin inhibits the growth of colorectal carcinoma by targeting tumor stem cells. Oncol Rep 2015, 34(5):2469-2476. 
8. Zhang C, Gong P, Liu P, Zhou N, Zhou Y, Wang Y: Thioridazine elicits potent antitumor effects in colorectal cancer stem cells. Oncol Rep 2017, 37(2):1168-1174.

9. Zhang C, Chen W, Zhang X, Zheng Y, Yu F, Liu Y, Wang Y: Grape seed proanthocyanidins induce mitochondrial pathway-mediated apoptosis in human colorectal carcinoma cells. Oncol Lett 2017, 14(5):5853-5860.

10. Visvader JE, Lindeman GJ: Cancer stem cells in solid tumours: accumulating evidence and unresolved questions. Nat Rev Cancer 2008, 8(10):755-768.

11. Zhou BBS, Zhang HY, Damelin M, Geles KG, Grindley JC, Dirks PB: Tumour-initiating cells: challenges and opportunities for anticancer drug discovery. Nat Rev Drug Discov 2009, 8(10):806-823.

12. Sarkar B, Dosch J, Simeone DM: Cancer Stem Cells: A New Theory Regarding a Timeless Disease. Chem Rev 2009, 109(7):3200-3208.

13. Mueller MT, Hermann PC, Witthauer J, Rubio-Viqueira B, Leicht SF, Huber S, Ellwart JW, Mustafa M, Bartenstein P, D'Haese JG et al: Combined Targeted Treatment to Eliminate Tumorigenic Cancer Stem Cells in Human Pancreatic Cancer. Gastroenterology 2009, 137(3):1102-1113.

14. Jeter CR, Badeaux M, Choy G, Chandra D, Patrawala L, Liu C, Calhoun-Davis T, Zaehres H, Daley GQ, Tang DG: Functional Evidence that the Self-Renewal Gene NANOG Regulates Human Tumor Development. Stem Cells 2009, 27(5):993-1005.

15. Lee M, Nam EJ, Kim SW, Kim S, Kim JH, Kim YT: Prognostic Impact of the Cancer Stem Cell-Related Marker NANOG in Ovarian Serous Carcinoma. Int J Gynecol Cancer 2012, 22(9):1489-1496.

16. Sun C, Sun L, Jiang K, Gao DM, Kang XN, Wang C, Zhang S, Huang S, Qin X, Li Y et al: NANOG promotes liver cancer cell invasion by inducing epithelial-mesenchymal transition through NODAL/SMAD3 signaling pathway. Int J Biochem Cell B 2013, 45(6):1099-1108.

17. Jeter CR, Liu B, Liu X, Chen X, Liu C, Calhoun-Davis T, Repass J, Zaehres H, Shen JJ, Tang DG: NANOG promotes cancer stem cell characteristics and prostate cancer resistance to androgen deprivation. Oncogene 2011, 30(36):3833-3845.

18. Yang L, Zhang XD, Zhang MZ, Zhang JH, Sheng YQ, Sun XD, Chen QJ, Wang LX: Increased Nanog Expression Promotes Tumor Development and Cisplatin Resistance in Human Esophageal Cancer Cells. Cell Physiol Biochem 2012, 30(4):943-952.

19. Zhang Y, Wang Z, Yu J, Shi JZ, Wang C, Fu WH, Chen ZW, Yang J: Cancer stem-like cells contribute to cisplatin resistance and progression in bladder cancer. Cancer Lett 2012, 322(1):70-77.

20. Wang P, Gao QL, Suo ZH, Munthe E, Solberg S, Ma LW, Wang MY, Westerdaal NAC, Kvalheim G, Gaudernack G: Identification and Characterization of Cells with Cancer Stem Cell Properties in Human Primary Lung Cancer Cell Lines. Plos One 2013, 8(3).

21. Meng HM, Zheng P, Wang XY, Liu C, Sui HM, Wu SJ, Zhou J, Ding YQ, Li JM: Overexpression of nanog predicts tumor progression and poor prognosis in colorectal cancer. Cancer Biol Ther 2010, 9(4):295302.

22. Fu C, Zhou N, Zhao Y, Duan J, Xu H, Wang Y: Dendritic cells loaded with CD44(+) CT-26 colon cell Iysate evoke potent antitumor immune responses. Oncol Lett 2019, 18(6):5897-5904. 
23. Ishiguro T, Sato A, Ohata H, Sakai H, Nakagama H, Okamoto K: Differential expression of nanog1 and nanogp8 in colon cancer cells. Biochem Biophys Res Commun 2012, 418(2):199-204.

24. Xu F, Dai CL, Zhang R, Zhao Y, Peng SL, Jia CJ: Nanog: A Potential Biomarker for Liver Metastasis of Colorectal Cancer. Digest Dis Sci 2012, 57(9):2340-2346.

25. Chu P, Clanton DJ, Snipas TS, Lee J, Mitchell E, Nguyen ML, Hare E, Peach RJ: Characterization of a subpopulation of colon cancer cells with stem cell-like properties. Int J Cancer 2009, 124(6):13121321.

26. Shaheen S, Ahmed M, Lorenzi F, Nateri AS: Spheroid-Formation (Colonosphere) Assay for in Vitro Assessment and Expansion of Stem Cells in Colon Cancer. Stem Cell Rev Rep 2016, 12(4):492-499.

27. Pan Q, Meng L, Ye J, Wei X, Shang Y, Tian Y, He Y, Peng Z, Chen L, Chen W et al: Transcriptional repression of miR-200 family members by Nanog in colon cancer cells induces epithelialmesenchymal transition (EMT). Cancer Lett 2017, 392:26-38.

28. Ibrahim EE, Babaei-Jadidi R, Saadeddin A, Spencer-Dene B, Hossaini S, Abuzinadah M, Li NN, Fadhil W, llyas M, Bonnet D et al: Embryonic NANOG Activity Defines Colorectal Cancer Stem Cells and Modulates through AP1-and TCF-dependent Mechanisms. Stem Cells 2012, 30(10):2076-2087.

29. Saiki Y, Ishimaru S, Mimori K, Takatsuno Y, Nagahara M, Ishii H, Yamada K, Mori M: Comprehensive Analysis of the Clinical Significance of Inducing Pluripotent Stemness-Related Gene Expression in Colorectal Cancer Cells. Ann Surg Oncol 2009, 16(9):2638-2644.

30. Galluzzi L, Brenner C, Morselli E, Touat Z, Kroemer G: Viral control of mitochondrial apoptosis. PLoS Pathog 2008, 4(5):e1000018.

31. Chen C, Ju R, Shi J, Chen W, Sun F, Zhu L, Li J, Zhang D, Ye C, Guo L: Carboxyamidotriazole Synergizes with Sorafenib to Combat Non-Small Cell Lung Cancer through Inhibition of NANOG and Aggravation of Apoptosis. J Pharmacol Exp Ther 2017, 362(2):219-229.

32. Ambady S, Malcuit C, Kashpur O, Kole D, Holmes WF, Hedblom E, Page RL, Dominko T: Expression of NANOG and NANOGP8 in a variety of undifferentiated and differentiated human cells. Int J Dev Biol 2010, 54(11-12):1743-1754.

33. Leibowitz B, Yu J: Mitochondrial signaling in cell death via the Bcl-2 family. Cancer Biol Ther 2010, 9(6):417-422.

34. Goodell MA, Brose K, Paradis G, Conner AS, Mulligan RC: Isolation and functional properties of murine hematopoietic stem cells that are replicating in vivo. J Exp Med 1996, 183(4):1797-1806.

35. Lee S, Schmitt CA: Chemotherapy response and resistance. Curr Opin Genet Dev 2003, 13(1):90-96.

36. Anwar TE, Kleer CG: Tissue-based identification of stem cells and epithelial-to-mesenchymal transition in breast cancer. Hum Pathol 2013, 44(8):1457-1464.

37. Lu H, Cao X, Zhang H, Sun G, Fan G, Chen L, Wang S: Imbalance between MMP-2, 9 and TIMP-1 promote the invasion and metastasis of renal cell carcinoma via SKP2 signaling pathways. Tumour Biol 2014, 35(10):9807-9813. 
38. Zajkowska M, Gacuta E, Kozlowska S, Lubowicka E, Glazewska EK, Chrostek L, Szmitkowski M, Pawlowski P, Zbucka-Kretowska M, Lawicki S: Diagnostic power of VEGF, MMP-9 and TIMP-1 in patients with breast cancer. A multivariate statistical analysis with ROC curve. Adv Med Sci 2019, 64(1):1-8.

39. Lee HJ, Noh KH, Lee YH, Song KH, Oh SJ, Kim SY, Kim TW: NANOG signaling promotes metastatic capability of immunoedited tumor cells. Clin Exp Metastasis 2015, 32(5):429-439.

\section{Table}

Table 1. Primers used for qRT-PCR

\begin{tabular}{lll} 
Gene & Forward primer $\mathbf{5}^{\prime} \rightarrow \mathbf{3}^{\prime}$ & Reverse primer $\mathbf{5}^{\mathbf{} \rightarrow \mathbf{3}^{\prime}}$ \\
\hline Nanog & GCCTCACACGGAGACTGTCTCTC & TTCTGGAACCAGGTCTTCACCTG \\
\hline Caspase-3 & CAGAACTGGACTGTGGCATTGAG & GGATGAACCAGGAGCCATCCT \\
\hline Bax & TAACCAAGGTGCCGGAACTGA & GGGAGGAGTCTCACCCAACCA \\
\hline Bal-2 & GGGGAGGATTGTGGCCTTCTTT & TAATGTGCAGGTGCCGGTTCAG \\
\hline MMP-2 & TCGCCCATCATCAAGTTCCC & TCTGGGGCAGTCCAAAGAAC \\
\hline MMP-9 & GCACCACCACAACATCACCT & ACCACAACTCGTCATCGTCG \\
\hline Timp-1 & TCGTCATCAGGGCCAAGTTC & TCCTGCAGTTTTCCAGCAATG \\
\hline Oct-4 & GCAGCTTGGGCTCGAGAAGGAT & AGCCCAGAGTGGTGACGGAGAC \\
\hline C-myc & CACCAGCAGCGACTCTGAGGAG & ACTTGACCCTCTTGGCAGCAGG \\
\hline GAPDH & CATCAGCAATGCCTCCTGCAC & TGAGTCCTTCCACGATACCAAAGTT
\end{tabular}

\section{Figures}


A
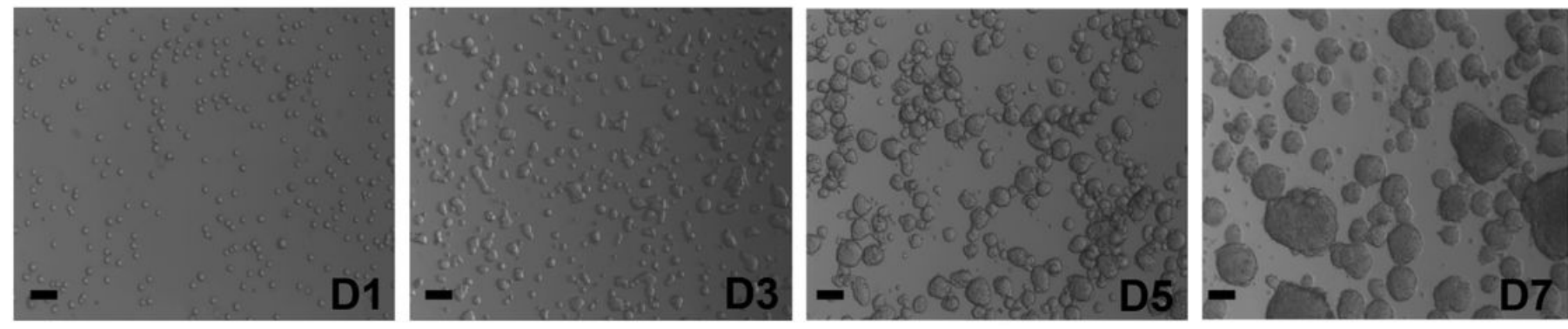

$\mathrm{B}$

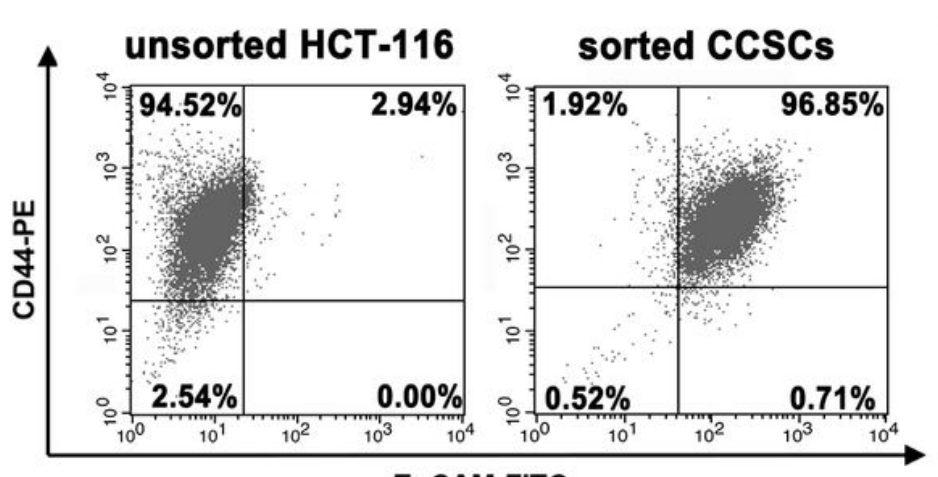

EpCAM-FITC

D
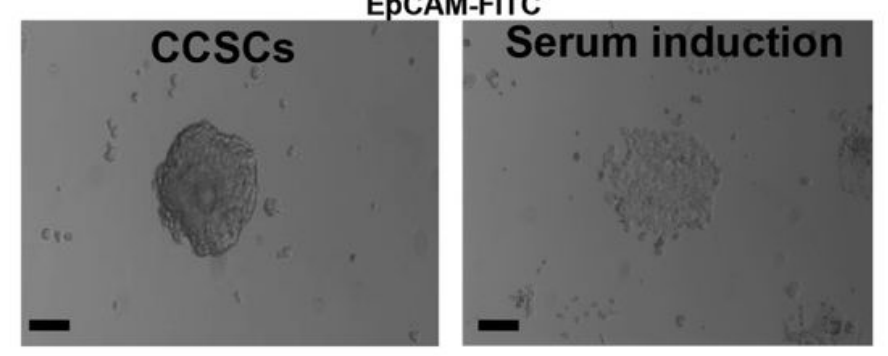

C

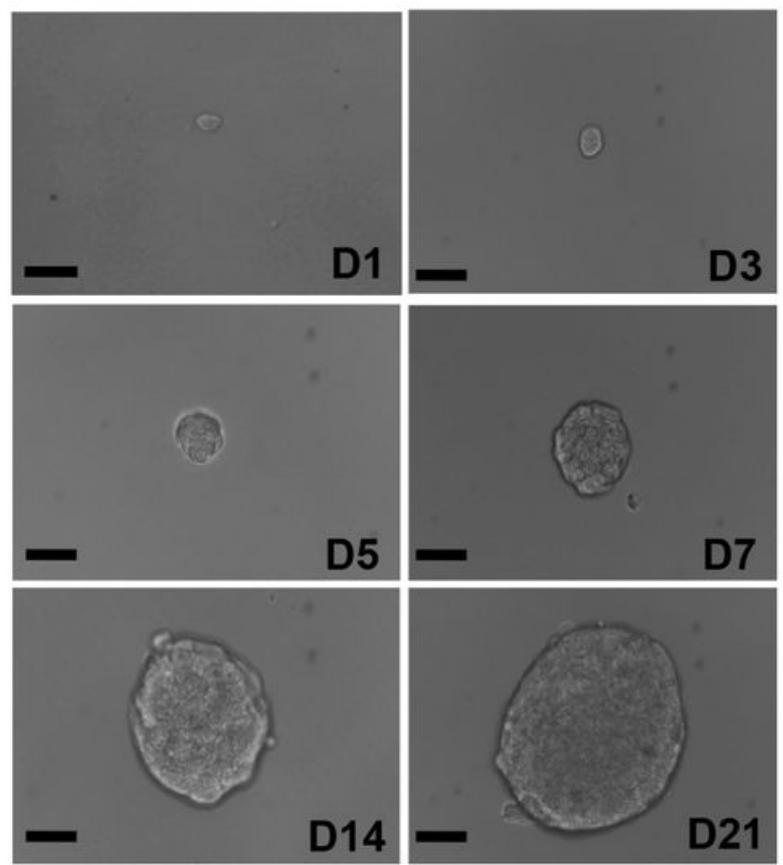

Figure 1

Optical micrographs and identification of sorted EpCAM+CD44+HCT-116 colorectal cancer stem cells (CCSCs). (A) Culture of CCSCs in serum-free DMEM/F12 medium over 7 days. (B) Flow cytometric analysis showing EpCAM and CD44 surface expression in CCSCs and unsorted HCT-116 cells. The majority of CCSCs (96.85\%) exhibited EpCAM+CD44+ staining, which was significantly higher than the percentage of unsorted HCT-116 cells (2.94\%). (C) Formation of CCSC spheres over 21 days analyzed by single-cell colony formation assay. (D) Serum-induced differentiation of CCSCs into adherent cells. (Scale bar: $200 \mu \mathrm{m})$ 


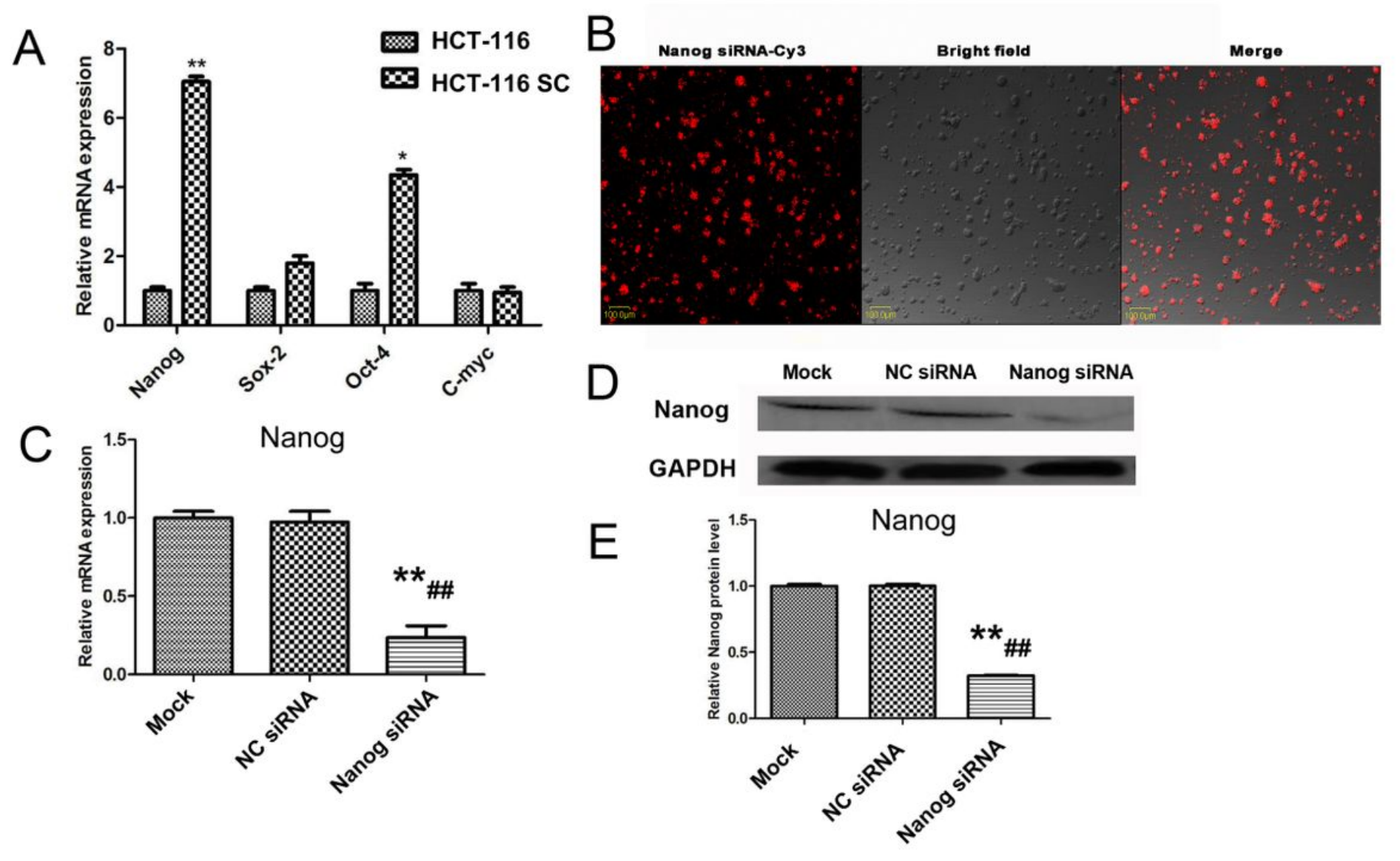

Figure 2

High expression level of Nanog in CCSCs and siRNA-mediated down-regulation of Nanog expression. (A) Real-time qPCR analysis showing relative mRNA expression level of Nanog in CCSCs compared with that in HCT-116 cells ( $\left.{ }^{*} P<0.05\right)$. (B) Fluorescent micrographs showing the transfection efficiency of Nanog siRNA. Representative fluorescence images of cy3-linked siRNA (red) are shown. (C) Real-time qPCR analysis showing Nanog mRNA levels in CSCs $48 \mathrm{~h}$ after siRNA transfection. (D, E) Western blot analysis showing Nanog protein expression levels following siRNA transfection of CCSCs. Data represent the means $\pm S D$ of three independent experiments ( ${ }^{\star * P}<0.01$, compared with mock; \#\#P<0.01, compared with negative-control siRNA). 


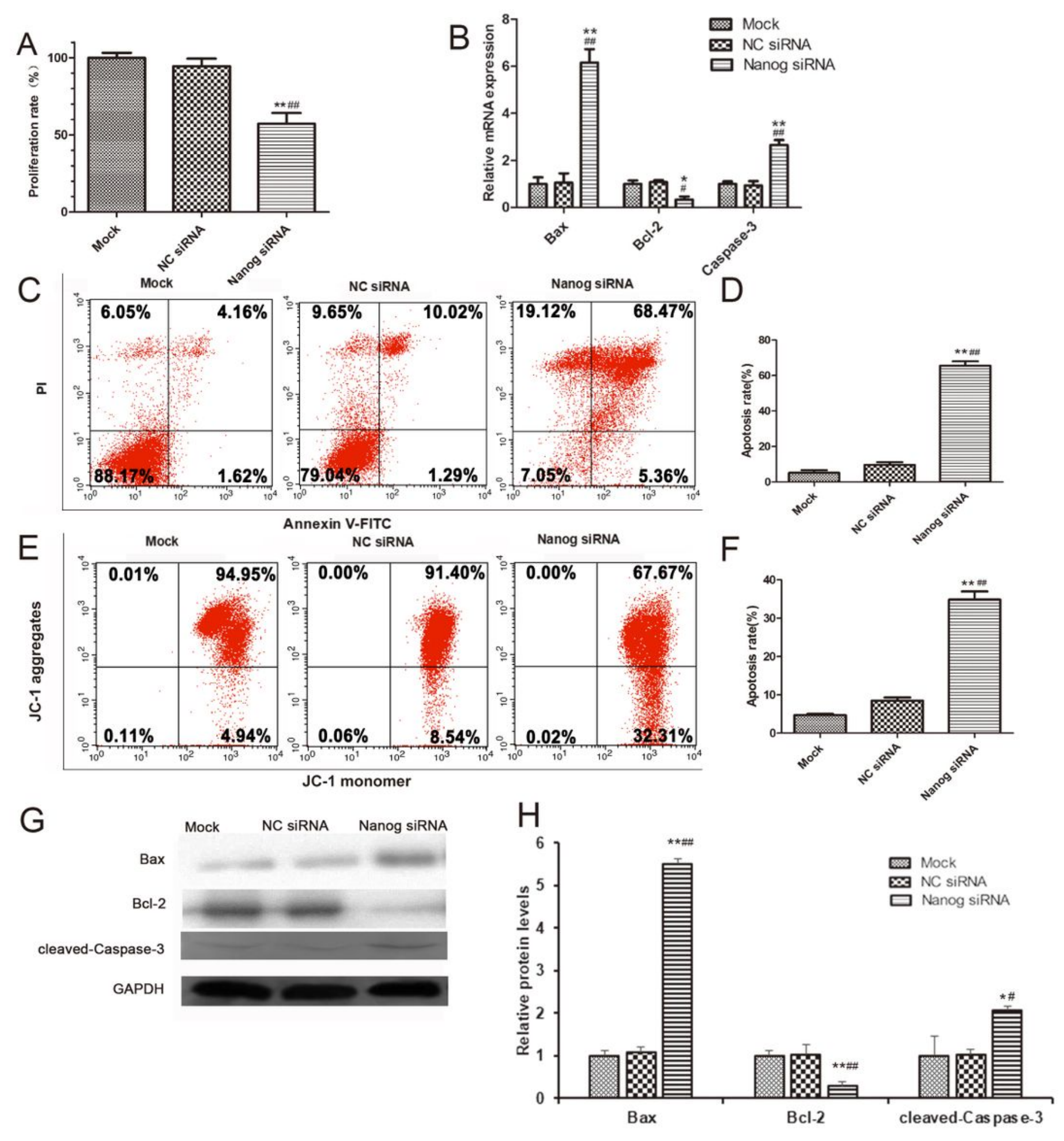

\section{Figure 3}

. Knockdown of Nanog expression suppresses CCSCs proliferation and induces apoptosis. (A) The MTS cell proliferation assay showing the proliferation potential of CCSCs $48 \mathrm{~h}$ after Nanog or control siRNA transfection. (B) Real-time qPCR analysis showing the mRNA levels of Bcl-2, Bax and caspase-3 in CCSCs after transfection with Nanog or control siRNA for 48 h. (C) Flow cytometric analyses of Annexin $\mathrm{V} /$ propidium iodide $(\mathrm{PI})$ staining of CCSCs following Nanog or control siRNA transfection for $48 \mathrm{~h}$. Lower- 
left quadrant, viable cells; upper-right and lower-right quadrants, apoptotic cells; upper-left quadrant, necrotic cells. (D) Annexin $V$ assay results showing the percentage of apoptotic CCSCs after treatment with Nanog or control siRNA. (E) Flow cytometric analysis of JC-1 staining of CCSCs after treatment with Nanog or control siRNA for $48 \mathrm{~h}$. Lower-right quadrant, apoptotic cells. (F) JC-1 assay results showing percentage of apoptotic CCSCs after treatment with Nanog or control siRNA $(G, H)$ Western blot analysis of the protein expression level of Bax, Bcl-2, and cleaved caspase-3 in CCSCs after treatment with Nanog or control siRNA for $48 \mathrm{~h}$. Data represent the means \pm SD of three independent experiments. ${ }^{*} P<0.05$ and $\star \star P<0.01$, compared with mock; $\# \mathrm{P}<0.05$ and \#\#P<0.01, compared with negative control siRNA.
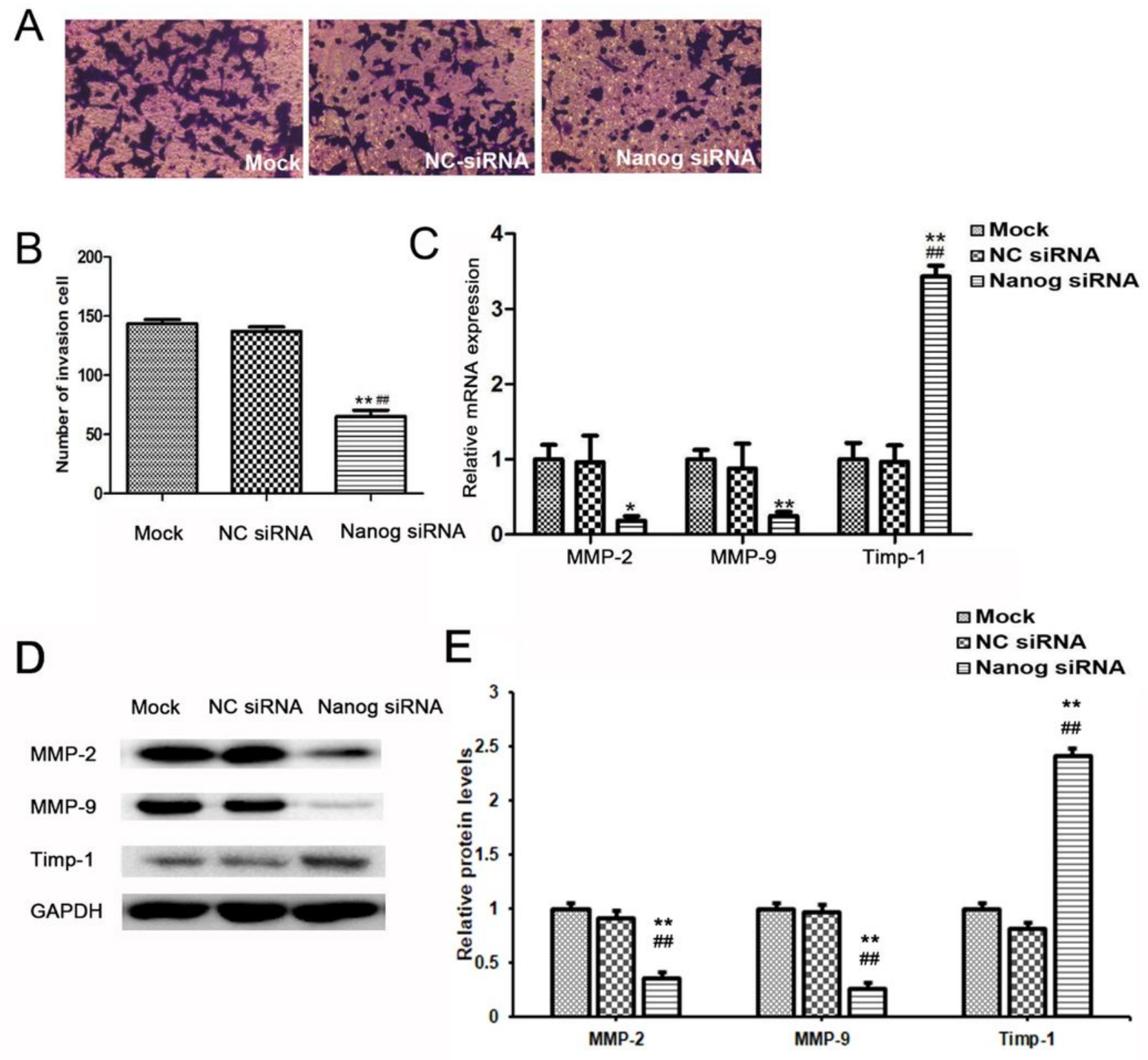

Figure 4

. Nanog silencing inhibits the invasive ability of CSCCs in vitro. (A) Representative photographs of cells passed through the matrigel in the Transwell invasion assay. (B) Quantification of the invasion assay 
indicating the number of invaded CCSCs. (C) Real-time qPCR analysis showing the relative mRNA levels of invasion-related genes, MMP-2, MMP-9, and TIMP-1 in CCSCs after treatment with Nanog or control siRNA for $48 \mathrm{~h}$. Data represent the means \pm SD of three independent experiments. ${ }^{*} \mathrm{P}<0.05$ and ${ }^{* \star} \mathrm{P}<$ 0.01 , compared with mock; \#\#P<0.01, compared with negative control siRNA.

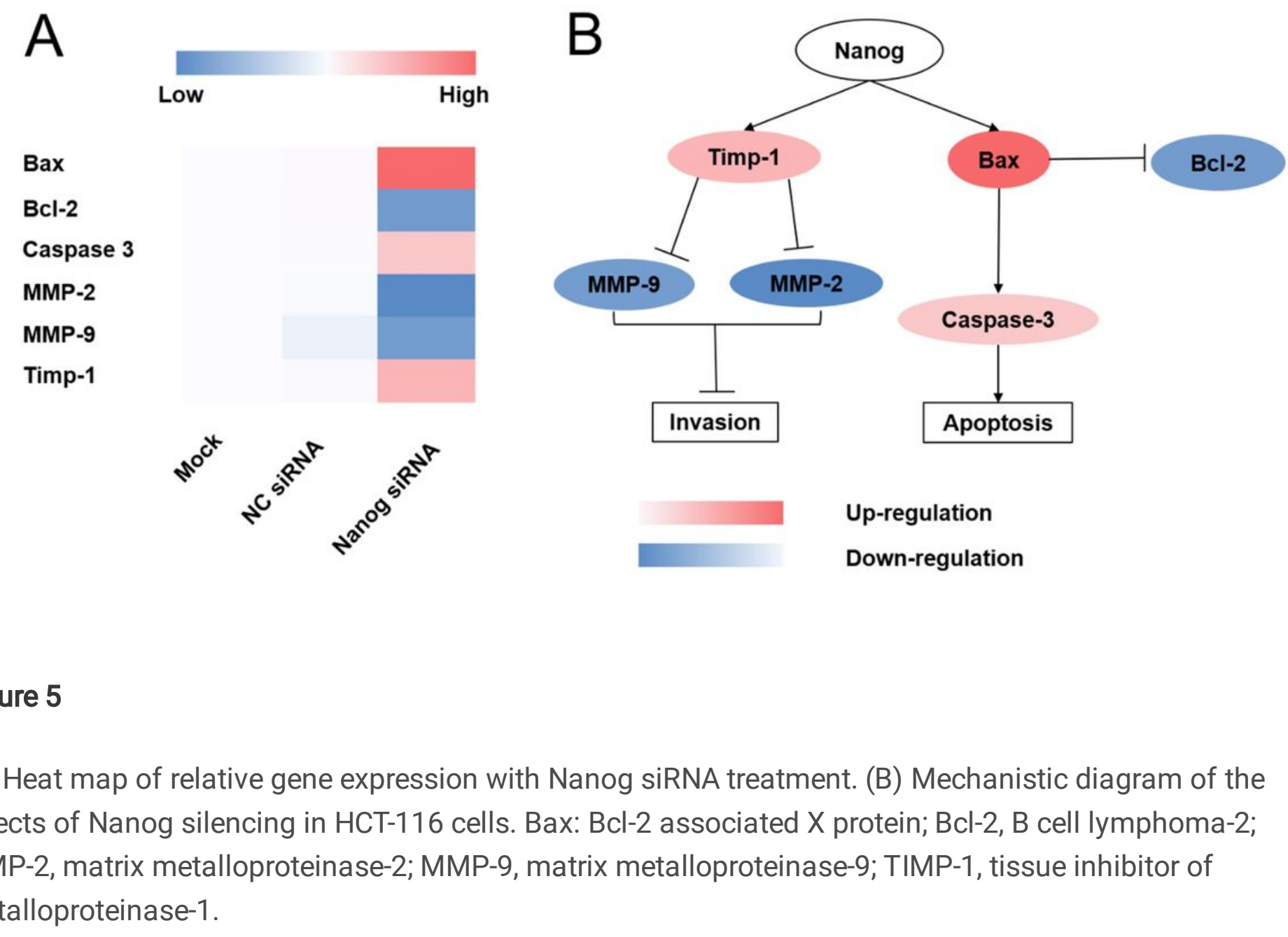




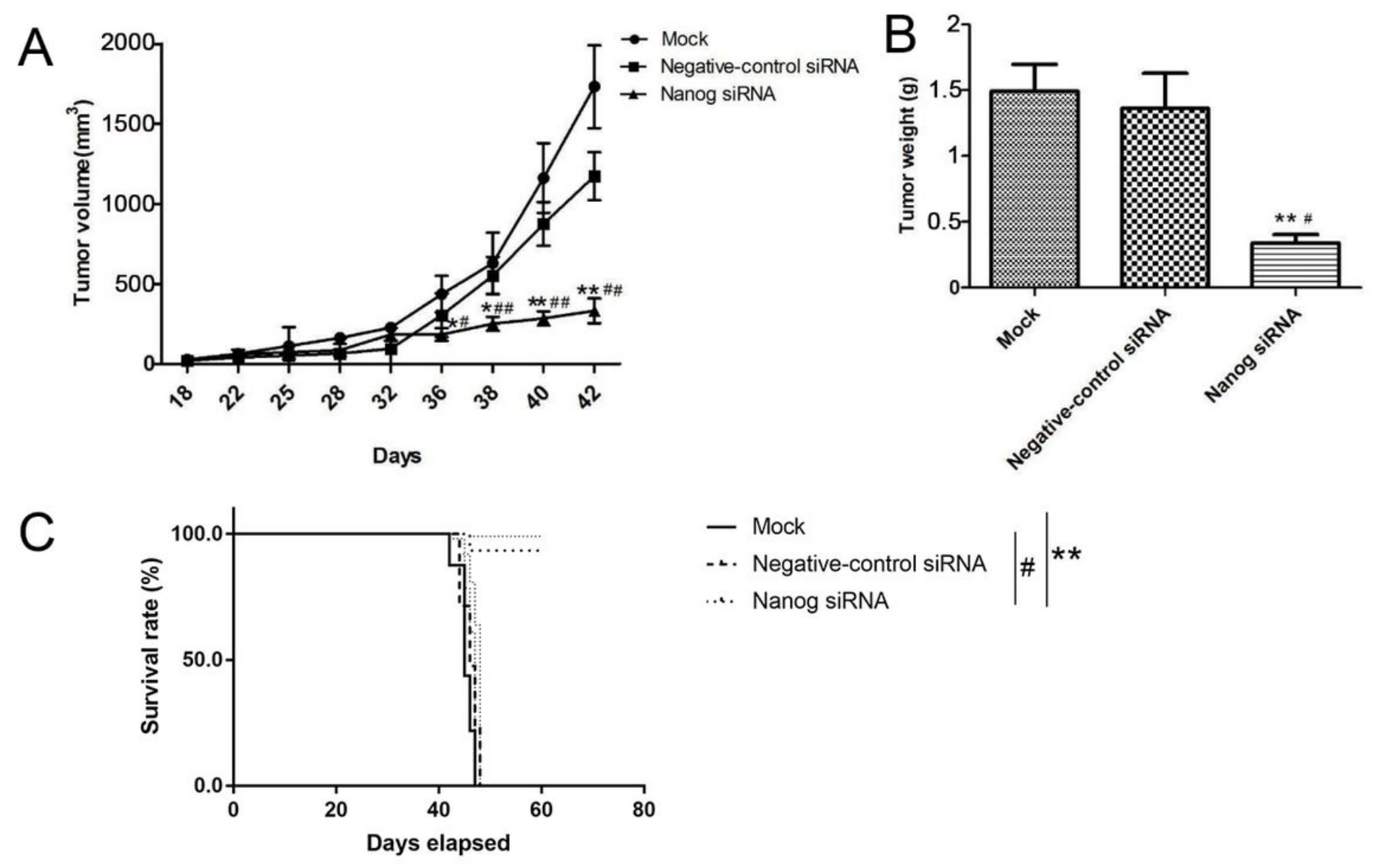

Figure 6

Nanog siRNA treatment suppressed xenograft tumor growth and prolonged mice survival. (A) Tumor volume and (B) tumor weight in the CCSCs xenograft mice following Nanog or negative control siRNA treatments for three weeks. (C) GraphPad prism 5 showing CCSCs xenograft mice survival after Nanog siRNA treatment. Data represent the means $\pm S D$. ${ }^{*} P<0.05$ and ${ }^{*} \mathrm{P}<0.01$, compared with mock; $\# \mathrm{P}<0.05$ and \#\#P<0.01 compared with negative control siRNA. 